\title{
Value and Limitations of Target-Vessel Ischemia in Predicting Late Clinical Events After Drug-Eluting Stent Implantation
}

\author{
Michael J. Zellweger ${ }^{1}$, Christoph Kaiser ${ }^{1}$, Hans Peter Brunner-La Rocca ${ }^{1}$, Peter T. Buser ${ }^{1}$, Stefan Osswald ${ }^{1}$, \\ Philipp Weiss ${ }^{1}$, Jan Mueller-Brand ${ }^{2}$, and Matthias E. Pfisterer ${ }^{1}$ for the BASKET Investigators \\ ${ }^{1}$ Department of Cardiology, University Hospital, Basel, Switzerland; and ${ }^{2}$ Department of Nuclear Medicine, University Hospital, \\ Basel, Switzerland
}

\begin{abstract}
Drug-eluting stents reduce clinical events related to restenosis but may be complicated by late stent-thrombosis. Whereas assessment of target-vessel ischemia by myocardial perfusion scintigraphy identifies relevant restenosis noninvasively, it is unknown whether this technique may also predict late clinical events related to late stent-thrombosis and to restenosis after drug-eluting stent implantation. Methods: All 826 patients treated with stenting between May 2003 and May 2004 were included in the Basel Stent Cost Effectiveness Trial (Basel Stent KostenEffektivitäts Trial, or BASKET) and randomized (2:1) to drugeluting stents or bare metal stents. Myocardial scintigraphy was performed on 476 (64\%) of 747 patients without major events after 6 mo. Patients were followed for 1 y for cardiac death, nonfatal myocardial infarction, and target-vessel revascularization due to restenosis or late stent-thrombosis. Results: The rate of target-vessel ischemia in these patients was lower with drug-eluting stents than with bare metal stents $(5.4 \%$ vs. $10.4 \%, P=0.045)$, similar to the rates of symptom-driven target-vessel revascularization up to $6 \mathrm{mo}(4.6 \%$ vs. $7.8 \%, P=$ 0.08 ). Ischemia was silent in $68 \%$. During follow-up, patients with target-vessel ischemia had higher event rates than did patients without ischemia ( $32.4 \%$ vs. $6.1 \%, P<0.001)$; however, ischemia did not predict late stent-thrombosis ( $0 / 11$ cases). Conclusion: The rate of clinical restenosis assessed scintigraphically was lower with drug-eluting stents than with bare metal stents and paralleled that of symptom-driven target-vessel revascularization. Target-vessel ischemia independently predicted late clinical events related to restenosis but not to late stent-thrombosis.
\end{abstract}

Key Words: coronary artery disease; revascularization; stent; restenosis; late stent-thrombosis; prognosis

J Nucl Med 2008; 49:550-556

DOI: 10.2967/jnumed.107.046771

\footnotetext{
Received Aug. 27, 2007; revision accepted Dec. 27, 2007.

For correspondence or reprints contact: Matthias Pfisterer, MD, Department of Cardiology, University Hospital, Petersgraben 4, CH-4031 Basel, Switzerland.

E-mail: pfisterer@email.ch

COPYRIGHT @ 2008 by the Society of Nuclear Medicine, Inc.
}

D rug-eluting stents reduce angiographic restenosis significantly $(1,2)$; however, late clinical events such as cardiac death and myocardial infarction (MI) limit the widespread acceptance of drug-eluting stent implantation. These late events may be due to restenosis $(3,4)$ or, as recently recognized, to late stent-thrombosis $(5,6)$. In identifying patients at risk for such late clinical events, noninvasive stress myocardial perfusion SPECT (MPS) may be useful, although firm evidence of usefulness has yet to be produced. Stress MPS has been shown to define clinically relevant restenosis in patients with or without symptoms (7-9). Also shown was that target-vessel ischemia was less prevalent after percutaneous coronary intervention (PCI) with stenting than without stenting (10). However, there are no data on the use of MPS in the drug-eluting stent era, in which restenosis rates may be so low that noninvasive testing may no longer be justified. In addition, the prognostic power of target-vessel ischemia to predict late clinical events has not been addressed specifically, nor has whether target-vessel ischemia may predict late events related to late stent-thrombosis. The aim of the present study was, first, to compare the rate of target-vessel ischemia after placement of drug-eluting stents with that after placement of bare metal stents in a large prospective trial of a consecutive group of unselected patients without major symptoms or events after $6 \mathrm{mo}$; second, to compare patients with target-vessel ischemia with those experiencing major adverse cardiac events (MACE) within $6 \mathrm{mo}$; and third, to assess the prognostic impact of target-vessel ischemia on late clinical events related to restenosis and to late stentthrombosis occurring over a further follow-up of $12 \mathrm{mo}$.

\section{MATERIALS AND METHODS}

\section{Design, Setting, and Patients}

All patients treated with PCI and stenting at the University Hospital of Basel, Switzerland, between May 5, 2003, and May 31,2004 , were included in the prospective randomized Basel Stent Cost Effectiveness Trial (Basel Stent Kosten-Effektivitäts Trial, or BASKET), irrespective of the indication for PCI, as previously reported (11). The only exclusion criteria were a target-vessel 
diameter of more than $4 \mathrm{~mm}$ (because the largest drug-eluting stent size available was $3.5 \mathrm{~mm}, n=23$ ), the presence of restenotic lesions (different etiology and outcome of restenotic lesions, $n=$ 49), and no consent (mostly because of patients' or referring physicians' preference for drug-eluting stents before angiography or because patients were unable to give informed consent, $n=90$ ), resulting in a patient population of 826 otherwise unselected patients. Seventy-three patients experienced MACE, and 6 died from noncardiac causes within the first 6 mo of the study. All 747 remaining patients with no or only mild to moderate symptoms 6 mo after the intervention were asked to undergo stress MPS, and 476 (64\%) consented. They formed the study population (Fig. 1) and gave informed written consent to this study, which was approved by the Ethics Committee of the States of Basel, Switzerland.

The representativeness and relevance of the findings were analyzed further through additional comparisons: patients with MPS versus those without, patients with MPS and target-vessel ischemia versus those with MPS and MACE during the 6 initial months of follow-up, and patients with MPS and target-vessel ischemia versus with those with MPS and no target-vessel ischemia (Fig. 1). All patients with stress MPS were followed for another year for prespecified clinical endpoints.

\section{Baseline Interventions and Concomitant Therapy}

PCI and stenting were performed according to standard procedures $(12,13)$, with the final decisions on the appropriate strategy in each patient left to the discretion of the operators in charge. In patients with ST-elevation MI, primary PCI was the treatment of choice. Patients with non-ST-elevation MI or unstable angina were treated urgently within $24 \mathrm{~h}$ of chest pain if possible, mostly with glycoprotein IIb/IIIa blocker therapy. Patients were randomized 2:1 to drug-eluting stents (Cypher Cordis; Johnson \& Johnson, or Taxus; Boston Scientific Corp.) or bare metal stents (Vision, a thirdgeneration cobalt-chromium stent; Guidant Corp.).

All patients were treated with aspirin $(100 \mathrm{mg} / \mathrm{d})$ and clopidogrel (300 mg periprocedurally; maintenance dose, $75 \mathrm{mg} / \mathrm{d}$ ) for $6 \mathrm{mo}$ irrespective of the type of stent used, and then clopidogrel was stopped but aspirin was continued long-term. Otherwise, the patients received the usual standard of care - that is, treatment with a statin and other drugs, including glycoprotein IIb/IIIa blockers, beta-blocking drugs, or angiotensin inhibitors as clinically indicated.

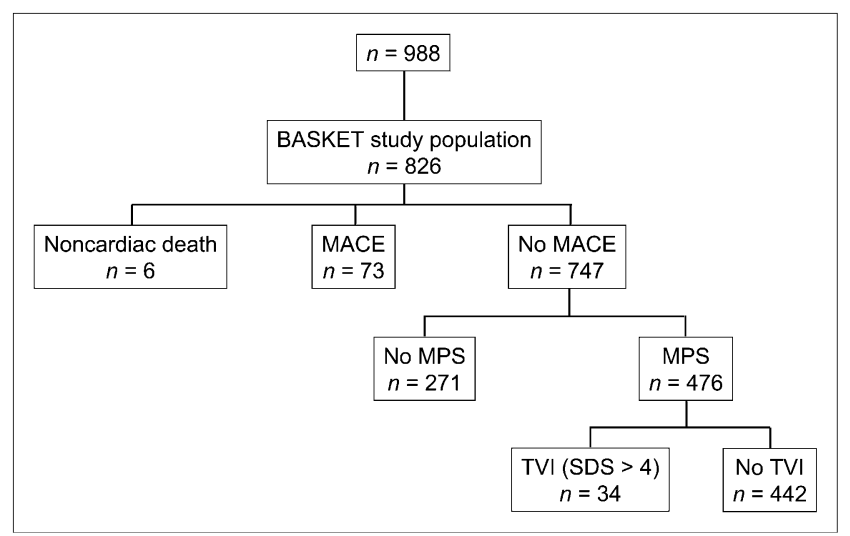

FIGURE 1. Study population: patient flow chart. TVI = targetvessel ischemia; SDS = summed difference score.

\section{Stress MPS}

For MPS, a rest-stress dual-isotope $\left({ }^{201} \mathrm{Tl}\right.$ rest/99m $\mathrm{Tc}$ sestamibi) protocol as previously described (14) was used. In patients not able to exercise or to reach an adequate stress test result, pharmacologic stress testing alone or, most frequently, in combination with mild exercise was used (33\% of the patient population underwent adenosine stress or combined testing).

Rest SPECT was performed after administration of $111 \mathrm{MBq}$ of ${ }^{201} \mathrm{Tl} .{ }^{201} \mathrm{Tl}$ SPECT was performed $10 \mathrm{~min}$ after tracer injection. A symptom-limited exercise test was performed, using routine protocols with a 12-lead electrocardiogram recording each minute of exercise and continuous monitoring of the electrocardiogram throughout the test. Whenever possible, beta-blocking medications and calcium antagonists were withheld for $48 \mathrm{~h}$, and long-acting nitrates for $24 \mathrm{~h}$, before exercise testing. The endpoints of exercise testing were physical exhaustion, severe angina, sustained ventricular arrhythmia, or exertional hypotension. At near-maximal exercise, a 740-MBq dose of ${ }^{99} \mathrm{~m}$ Tc-sestamibi was injected, and exercise was continued for at least an additional minute after injection. Blood pressure and the electrocardiogram were recorded according to current guidelines (15). In patients undergoing pharmacologic stress, adenosine was infused $(140 \mathrm{mg} / \mathrm{kg} / \mathrm{min}$ for $6 \mathrm{~min})$, and ${ }^{99 \mathrm{~m}} \mathrm{Tc}$ sestamibi was injected at the end of the third minute of infusion. Patients were instructed that, during the $24 \mathrm{~h}$ before the adenosine stress test, they were not to consume products containing caffeine. SPECT was performed by following standard protocols (16). No attenuation or scatter correction was used.

SPECT images were acquired and processed as previously described (17), with a circular $180^{\circ}$ acquisition. During imaging, 2 energy windows were used for ${ }^{201} \mathrm{Tl}$, including a $30 \%$ window centered on the $70-\mathrm{keV}$ peak and a $20 \%$ window centered on the $167-\mathrm{keV}$ peak. For ${ }^{99 \mathrm{~m}} \mathrm{Tc}$-sestamibi SPECT, a $15 \%$ window centered on the $140-\mathrm{keV}$ peak was used. Perfusion images were scored using a 20 -segment model with a 5-point scale $(0=$ normal, $1=$ mildly reduced tracer uptake, $2=$ moderately reduced uptake, $3=$ severely reduced uptake, and $4=$ no uptake). The overall summed stress score and summed rest score were calculated by adding the scores of the 20 segments in the stress and rest images, respectively. The overall summed difference score was derived as the difference between stress and rest scores as a measure of ischemia. If ischemia was present, we correlated the treated target vessel and ischemia on the basis of the segment distribution suggested in the scientific statement paper of the American Heart Association (18). If there was doubt about correspondence (e.g., inferolateral wall distribution of right coronary artery or left circumflex coronary artery), we correlated vessel distribution and perfusion by looking at the particular coronary anatomy as provided by coronary angiography. Target-vessel ischemia was defined as a matching of target-vessel distribution and ischemia. In addition, ischemia per se was defined as a summed difference score of more than 4 in the target vessel.

\section{Follow-up and Endpoint Definitions}

In accord with the BASKET protocol (11), all patients were prospectively evaluated on an outpatient basis after 6 mo for primary endpoint assessment. Patients were then followed for another $12 \mathrm{mo}$ for MACE as defined below, to define the prognostic impact of target-vessel ischemia (19). During the entire study, no "routine" repeated coronary angiographies were allowed if clinical symptoms were absent or only mild.

Prespecified major clinical endpoints (MACE) were cardiac death, nonfatal MI, and non-MI-related target-vessel revasculari- 
zation. Nonfatal MI apart from the index intervention was diagnosed on the basis of a typical rise (and fall) of cardiac enzymes, typical chest pain, and new pathologic Q-waves or ischemic ST-T wave changes in the electrocardiogram according to current guidelines. Repeated MI after an acute intervention was a clinical diagnosis based on new chest pain together with typical enzyme elevation or electrocardiogram changes. Target-vessel revascularization was defined as intervention (PCI or bypass surgery) driven by a lesion in the same epicardial vessel as that initially treated. Because targetvessel revascularization events related to MI may be clinically more significant than other target-vessel revascularizations and may have a different underlying mechanism, death and MI as hard endpoints and target-vessel revascularization not related to these hard endpoints (i.e., non-MI-related target-vessel revascularization) were considered separately. All events were adjudicated by an independent Critical Events Committee unaware of the stent type used. Events related to late stent-thrombosis were those with angiographic or autopsy documentation of stent thrombosis, as well as targetvessel MI occurring after $1 \mathrm{y}$ according to the definition of the Academic Research Consortium (5,20).

\section{Statistical Analysis}

Quantitative data are given as mean and SD or as median. Categoric data are described by their distribution. Group comparisons were performed with the Fisher exact test for categoric variables or with the unpaired $t$ test or Mann-Whitney $U$ test for quantitative variables. A multivariate logistic regression model was used to identify variables independently predictive of target-vessel ischemia. A forward stepwise method was applied for inclusion or exclusion into the model. Variables that were significantly different between patients with ischemia and patients without ischemia were incorporated into the model. Because the ischemia group had no patient with a type A lesion, that variable was not included.

Kaplan-Meier cumulative event curves were used to compare outcomes between patients with target-vessel ischemia and patients without (log-rank test). In addition, a Cox proportional hazards regression model was used to evaluate the independent predictors of MACE. A $P$ value of less than 0.05 was considered statistically significant.

\section{RESULTS}

\section{Selection of Patients for MPS}

The baseline characteristics of the 476 patients with follow-up MPS versus the 271 without are summarized in Table 1. Patients who did not consent to MPS were more likely to have been initially transferred from another hospital and had fewer stents implanted, for a shorter total stent length per patient, than did patients who consented to MPS. This finding indicates that patients cared for and followed at the University Hospital of Basel and those at slightly higher risk were more likely to undergo follow-up MPS than were patients referred from other hospitals. Importantly, patients who underwent MPS did not significantly differ in stent type received from those who did not undergo MPS.

\section{Incidence of Target-Vessel Ischemia}

Of the 476 patients who underwent MPS, 34 (7.1\%) had target-vessel ischemia as defined for this study. The baseline characteristics of patients with target-vessel ischemia versus patients without are shown in Table 2. Target-vessel

TABLE 1

Baseline Characteristics of Patients With Follow-up MPS versus Patients Without

\begin{tabular}{|c|c|c|c|}
\hline Characteristic & No MPS $(n=271)$ & $\operatorname{MPS}(n=476)$ & $P$ \\
\hline Female (\%) & 23 & 21 & 0.5 \\
\hline $\operatorname{Age}^{*}(y)$ & $63 \pm 11$ & $63 \pm 11$ & 0.9 \\
\hline Diabetes (\%) & 17 & 19 & 0.5 \\
\hline Hypercholesterolemia (\%) & 76 & 78 & 0.6 \\
\hline Hypertension (\%) & 66 & 67 & 0.9 \\
\hline Smoking (\%) & 27 & 30 & 0.4 \\
\hline Single-vessel (\%) & 31 & 35 & 0.4 \\
\hline Double-vessel (\%) & 33 & 33 & 0.4 \\
\hline Triple-vessel (\%) & 36 & 32 & 0.4 \\
\hline Left main (\%) & 0.7 & 1.3 & 0.5 \\
\hline Prior MI (\%) & 30 & 26 & 0.2 \\
\hline Acute ST-elevation MI (\%) & 24 & 20 & 0.3 \\
\hline Acute coronary syndrome (\%) & 36 & 37 & 0.3 \\
\hline Angina (\%) & 40 & 44 & 0.3 \\
\hline Referral from periphery (\%) & 42 & 34 & 0.02 \\
\hline Cypher stents (\%) & 32 & 34 & 0.2 \\
\hline Taxus stents (\%) & 38 & 32 & 0.2 \\
\hline Bare metal stents (\%) & 30 & 34 & 0.2 \\
\hline 2.5-mm stent only (\%) & 24 & 29 & 0.14 \\
\hline 3.5-mm stent only (\%) & 31 & 23 & 0.02 \\
\hline Number of stents per patient* & $1.4 \pm 0.6$ & $1.6 \pm 0.7$ & $<0.0001$ \\
\hline Total length of stents $(\mathrm{mm})$ per patient ${ }^{*}$ & $31 \pm 17$ & $35 \pm 22$ & 0.006 \\
\hline
\end{tabular}


TABLE 2

Baseline Characteristics of Patients With Target-Vessel Ischemia Versus Patients Without

\begin{tabular}{|c|c|c|c|}
\hline Characteristic & $\begin{array}{l}\text { No target-vessel ischemia } \\
\qquad(n=442)\end{array}$ & $\begin{array}{l}\text { Target-vessel ischemia } \\
\qquad(n=34)\end{array}$ & $P$ \\
\hline Female (\%) & 21 & 24 & 0.6 \\
\hline $\operatorname{Age}^{*}(y)$ & $63 \pm 11$ & $65 \pm 12$ & 0.4 \\
\hline Diabetes (\%) & 19 & 27 & 0.3 \\
\hline Hypercholesterolemia (\%) & 77 & 88 & 0.2 \\
\hline Hypertension (\%) & 67 & 68 & 1.0 \\
\hline Smoking (\%) & 30 & 18 & 0.2 \\
\hline Single-vessel (\%) & 35 & 24 & 0.2 \\
\hline Double-vessel (\%) & 34 & 21 & 0.1 \\
\hline Triple-vessel (\%) & 31 & 56 & 0.002 \\
\hline Left main (\%) & 1.1 & 2.9 & 0.4 \\
\hline Prior MI (\%) & 25 & 32 & 0.4 \\
\hline Acute ST-elevation MI (\%) & 20 & 18 & 0.9 \\
\hline Acute coronary syndrome (\%) & 37 & 41 & 0.9 \\
\hline Angina (\%) & 44 & 41 & 0.9 \\
\hline Referral from periphery (\%) & 34 & 32 & 0.9 \\
\hline Cypher stents (\%) & 34 & 24 & 0.2 \\
\hline Taxus stents (\%) & 33 & 27 & 0.4 \\
\hline Bare metal stents (\%) & 33 & 50 & 0.04 \\
\hline 2.5-mm stent only (\%) & 28 & 35 & 0.4 \\
\hline 3.5-mm stent only (\%) & 24 & 18 & 0.5 \\
\hline Number of stents per patient* & $1.6 \pm 0.7$ & $1.6 \pm 0.7$ & 0.9 \\
\hline Type A lesion (\%) & 17 & 0 & 0.008 \\
\hline Total length of stents $(\mathrm{mm})$ per patient ${ }^{*}$ & $35 \pm 22$ & $35 \pm 26$ & 0.9 \\
\hline Median overall summed stress score & 4 & 15 & $<0.0001$ \\
\hline Median overall summed rest score & 3 & 7 & 0.027 \\
\hline Median overall summed difference score & 0 & 7 & $<0.0001$ \\
\hline
\end{tabular}

ischemia rates were significantly lower with drug-eluting stents than with bare metal stents $(5.4 \%$ vs. $10.4 \%, P=$ 0.045; Fig. 2), a result that paralleled the findings for MACE in BASKET $(7.2 \%$ with drug-eluting stents vs. $12.1 \%$ with bare metal stents, $P=0.02$ ) and, particularly, the findings for symptom-driven target-vessel revasculari-

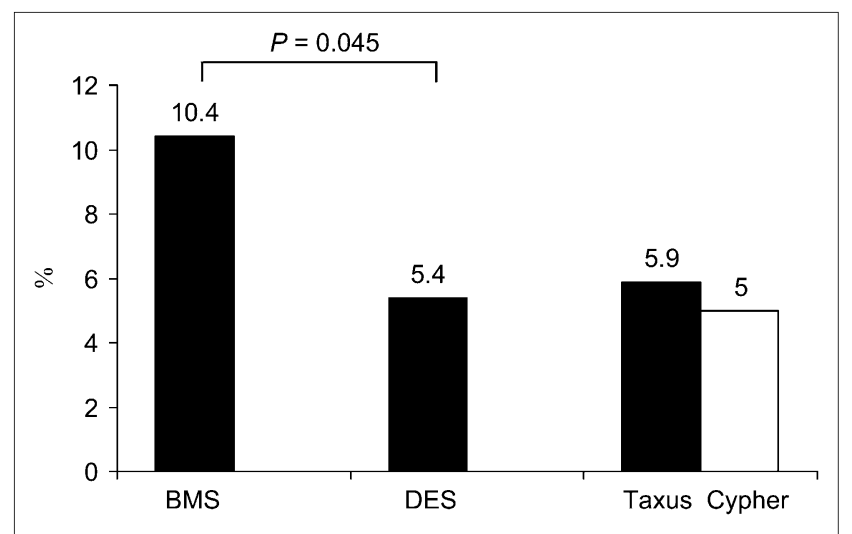

FIGURE 2. Target-vessel ischemia rates with respect to stent type implanted. BMS = bare metal stent; DES = drug-eluting stent. zation (4.6\% with drug-eluting stents vs. $7.8 \%$ with bare metal stents, $P=0.08)$. Of note, $23(68 \%)$ of the patients with target-vessel ischemia had no angina and therefore silent ischemia during the stress test. When typical angina during daily activities was evaluated, $32 \%$ of patients with target-vessel ischemia reported angina (26\% class II and $6 \%$ class III); thus, these patients had only mild symptoms that were well controlled by drug therapy.

\section{Relevance of Target-Vessel Ischemia}

Compared with the 73 patients with MACE within the first 6 mo, patients with target-vessel ischemia differed from these at baseline only with regard to the frequency of hypercholesterolemia, which was present in $88 \%$ of patients with target-vessel ischemia and $64 \%$ of patients with MACE $(P=0.02)$.

Patients with target-vessel ischemia had a higher prevalence of triple-vessel disease, a higher rate of bare metal stents, and fewer type A lesions than did patients without target-vessel ischemia. In a multivariate logistic regression analysis, only triple-vessel disease (odds ratio, 3.0; $P=$ 0.003 ) and drug-eluting stents (odds ratio, $0.46 ; P=0.035$ ) were independent predictors of target-vessel ischemia. $\mathrm{Pa}-$ tients with a type A lesion were not incorporated into this 
analysis because no patient in the target-vessel ischemia group had a type A lesion. Similarly, independent predictors of MACE up to 6 mo in BASKET were triple-vessel disease (odds ratio, 2.03; $P=0.006$ ), residual stenosis greater than $50 \%$ (odds ratio, 2.38; $P=0.02$ ), more than one treated segment (odds ratio, 1.69; $P=0.05$ ), and use of drug-eluting stents (odds ratio, $0.58 ; P=0.03$ ) (11).

\section{Prognostic Impact of Target-Vessel Ischemia}

During a follow-up of 12 mo after MPS, 15 patients (of $476 ; 3.2 \%)$ died of a cardiac cause $(n=3)$ or had a nonfatal MI $(n=12)$ and 32 patients $(6.7 \%)$ underwent target-vessel revascularization (for symptoms that were new, increasing, or severe), resulting in a total of $47 \mathrm{MACE}(9.9 \%)$. Late stentthrombosis was identified as the cause of these events in 11 (23\%) of 47 patients, for an overall rate of definite or probable late stent-thrombosis of 11 (2.3\%) of 476 patients within $1 \mathrm{y}$. Overall, patients with target-vessel ischemia at 6 mo had significantly more MACE than did those without targetvessel ischemia (32.4\% vs. $6.1 \%, P<0.001$; Fig. 3). Figure 4 shows that the rate of MACE is increasing as a function of the extent of overall ischemia (summed difference score) and that risk stratification by the extent of ischemia is possible. In Figure 5, the cumulative event curves between months 7 and 18 are shown in patients with target-vessel ischemia at 6 mo versus patients without. Patients with target-vessel ischemia had a significantly worse event-free survival than did patients without target-vessel ischemia, and this finding held true even if target-vessel revascularization events within the first 2 mo after MPS were disregarded because they might have been induced in part by MPS findings (28\% with targetvessel ischemia vs. $4.8 \%$ with no target-vessel ischemia, $P<$ $0.0001)$. Note again that angiography and target-vessel revascularization were allowed in BASKET only if patients were severely symptomatic despite medical therapy.

A Cox proportional hazards model (incorporating age, sex, summed stress score [overall abnormality of MPS findings], and target-vessel ischemia) revealed that target-vessel ischemia (hazard ratio, 3.6; 95\% confidence interval, 1.6-7.8; $P=$ 0.001 ) and summed stress score (hazard ratio, 1.06; $95 \%$

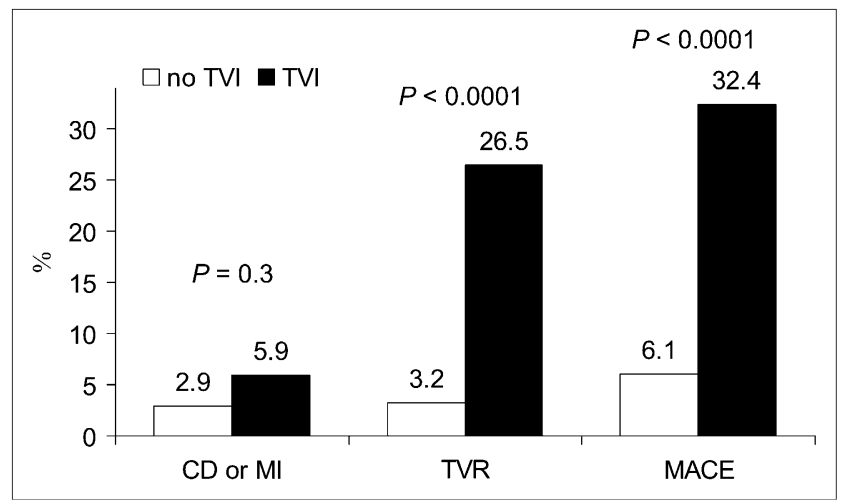

FIGURE 3. Event rates in patients with target-vessel ischemia (TVI) vs. patients without. $C D=$ cardiac death.

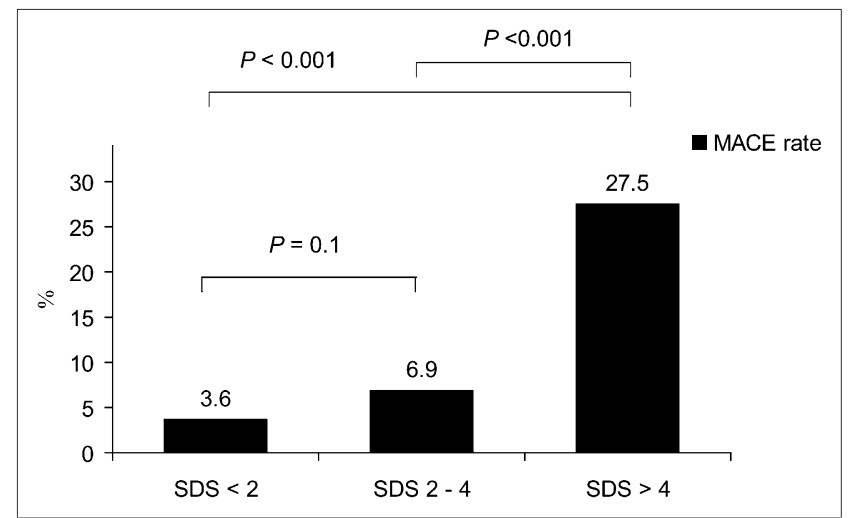

FIGURE 4. Rates for MACE as function of overall summed difference score (SDS).

confidence interval, $1.02-1.1 ; P=0.002)$ were independent predictors of MACE.

For comparison, of the remaining 271 patients without MPS or MACE, $10.0 \%$ experienced MACE during late follow-up, similar to the $9.9 \%$ of patients who underwent MPS. Of note, MPS predicted 11 of 36 restenosis-related events - that is, cardiac death, nonfatal MI, or target-vessel revascularization-but none of the 11 thrombosis-related events.

\section{DISCUSSION}

In contrast to previous trials on angiographic restenosis after PCI and stenting, the present study focused on clinically relevant restenosis defined by target-vessel ischemia as assessed by MPS. We have presented, for what is to our knowledge the first time, comparative data on clinical restenosis after stenting with drug-eluting stents and bare metal stents in unselected patients. The findings further confirm the superiority of drug-eluting stents over bare metal stents in this regard and are in close relation to 6-mo MACE, particularly target-vessel revascularization rates. In addition, the

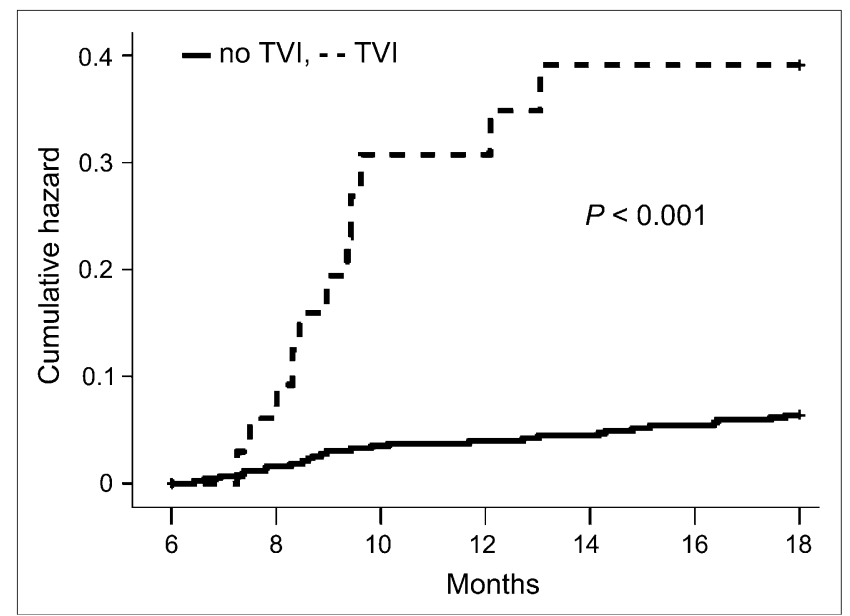

FIGURE 5. Rates for MACE in patients with target-vessel ischemia (TVI) vs. patients without. 
findings document the significance of target-vessel ischemia in the prediction of restenosis-related events. However, target-vessel ischemia failed to predict events related to late stent-thrombosis, suggesting a different pathophysiology for these different late clinical events - that is, intima hyperplasia as a cause of restenosis versus incomplete stent coverage (healing) as a cause of late stent-thrombosis.

The findings of target-vessel ischemia are clinically relevant because they predicted clinical events independently during further follow-up. Importantly, these events were not biased by an "oculo-stenotic reflex" that may have influenced prior studies with protocol-driven angiographic follow-up, because repeated revascularization was allowed only in severely symptomatic patients. Therefore, target-vessel ischemia findings in the subgroup of patients with no or only mild to moderate angina did not prompt repeated revascularization in BASKET-Late Thrombotic Events $(11,19)$. This observation is underscored by the finding that the exclusion of events within 2 mo after MPS did not influence the results. The finding that the presence of target-vessel ischemia is associated with a 5-fold increase in late MACE, compared with the rate of late MACE in the absence of targetvessel ischemia, points to the strong predictive power of MPS. These results reinforce earlier findings after balloon angioplasty (9) or after bare metal stenting (8), as well as the high rate of asymptomatic ischemia in this situation, and extend them to the era of drug-eluting stents. The clinical relevance of target-vessel ischemia is also stressed by the similar and parallel findings of target-vessel revascularization and MACE (11). In addition, the doubling of the rate of hard events, that is, cardiac death or nonfatal MI, in patients with target-vessel ischemia versus those without (Fig. 3) supports the suggestion that target-vessel ischemia, or restenosis, is not an entirely benign finding. Although targetvessel ischemia rates for bare metal stents were relatively low in patients without clinical events within the first $6 \mathrm{mo}$, target-vessel ischemia rates were significantly lower in patients treated with drug-eluting stents. Target-vessel ischemia rates for bare metal stents were similar to rates previously reported for stainless-steel bare metal stents: 8\%-10\% (7).

Importantly, however, this study led to another relevant clinical observation: Although target-vessel ischemia predicted late events, it predicted those related to restenosis but not late events related to late stent-thrombosis. This finding points to different mechanisms underlying late clinical events after drug-eluting stent implantation: clinical events due to restenosis on the one hand, which may be predicted by target-vessel ischemia, and clinical events due to late stentthromboses on the other hand, which seem to occur in the absence of target-vessel ischemia or restenosis as new, sudden, and therefore "unpredictable" events.

A limitation of this study is that not all patients underwent 6-mo MPS and that a power calculation was performed only for the overall BASKET trial. This limitation also holds true for many previous studies on restenosis with angiographic follow-up in which a considerable percentage of patients refused repeated coronary angiography. Therefore, we compared the study group carefully with patients who did not undergo MPS, as well as comparing patients with targetvessel ischemia with those who experienced MACE within the initial $6 \mathrm{mo}$, which showed minor differences only. Importantly, the rate of MACE in patients with MPS was identical to that in patients without MPS. Thus, one may assume that the present findings are valid for a general patient population after PCI and stenting. Because not all patients with MPS underwent coronary angiography, we cannot exclude the possibility that progression of disease may also have affected target-vessel ischemia findings. Although the target vessel was treated at baseline with the aim of full revascularization, and the 6-mo period seems too short for disease progression to have had a major impact on the presented findings, we cannot exclude disease progression entirely.

\section{CONCLUSION}

In patients with mild to moderate or no symptoms after stenting, target-vessel ischemia proved to be a relevant clinical marker of restenosis that was significantly lower after implantation of drug-eluting stents than after implantation of bare metal stents and paralleled the findings for MACE and, particularly, symptom-driven target-vessel revascularization. In two thirds of patients, target-vessel ischemia was not associated with chest pain. During the following year, 6-mo target-vessel ischemia strongly predicted clinical events related to restenosis but could not predict events related to late stent-thrombosis, pointing to different etiologies for these late clinical events.

\section{ACKNOWLEDGMENT}

We acknowledge the BASKET investigators, a list of whom has been previously published by Kaiser et al. (11). This study was supported by the Basel Cardiovascular Research Foundation, Basel, Switzerland.

\section{REFERENCES}

1. Spaulding C, Daemen J, Boersma E, Cutlip DE, Serruys PW. A pooled analysis of data comparing sirolimus-eluting stents with bare-metal stents. N Engl J Med. 2007;356:989-997.

2. Kastrati A, Mehilli J, Pache J, et al. Analysis of 14 trials comparing sirolimuseluting stents with bare-metal stents. N Engl J Med. 2007;356:1030-1039.

3. Indolfi C, Pavia M, Angelillo IF. Drug-eluting stents versus bare metal stents in percutaneous coronary interventions (a meta-analysis). Am J Cardiol. 2005;95: 1146-1152.

4. Serruys PW, Kutryk MJ, Ong AT. Coronary-artery stents. $N$ Engl J Med. 2006;354:483-495.

5. Mauri L, Hsieh WH, Massaro JM, Ho KK, D'Agostino R, Cutlip DE. Stent thrombosis in randomized clinical trials of drug-eluting stents. $N$ Engl J Med. 2007;356:1020-1029.

6. Stone GW, Moses JW, Ellis SG, et al. Safety and efficacy of sirolimus- and paclitaxel-eluting coronary stents. N Engl J Med. 2007;356:998-1008.

7. Zellweger MJ, Weinbacher M, Zutter AW, et al. Long-term outcome of patients with silent versus symptomatic ischemia six months after percutaneous coronary intervention and stenting. J Am Coll Cardiol. 2003;42:33-40. 
8. Pfisterer M, Rickenbacher P, Kiowski W, Muller-Brand J, Burkart F. Silent ischemia after percutaneous transluminal coronary angioplasty: incidence and prognostic significance. J Am Coll Cardiol. 1993;22:1446-1454.

9. Giedd KN, Bergmann SR. Myocardial perfusion imaging following percutaneous coronary intervention: the importance of restenosis, disease progression, and directed reintervention. J Am Coll Cardiol. 2004;43:328-336.

10. Ruygrok PN, Webster MW, de Valk V, et al. Clinical and angiographic factors associated with asymptomatic restenosis after percutaneous coronary intervention. Circulation. 2001;104:2289-2294.

11. Kaiser C, Brunner-La Rocca HP, Buser PT, et al. Incremental cost-effectiveness of drug-eluting stents compared with a third-generation bare-metal stent in a real-world setting: randomised Basel Stent Kosten Effektivitats Trial (BASKET). Lancet. 2005;366:921-929.

12. Smith SC Jr, Feldman TE, Hirshfeld JW Jr, et al. ACC/AHA/SCAI 2005 Guideline Update for Percutaneous Coronary Intervention: summary article-a report of the American College of Cardiology/American Heart Association Task Force on Practice Guidelines (ACC/AHA/SCAI writing committee to update the 2001 guidelines for percutaneous coronary intervention). Circulation. 2006;113: 156-175.

13. Smith SC Jr, Dove JT, Jacobs AK, et al. ACC/AHA guidelines for percutaneous coronary intervention (revision of the 1993 PTCA guidelines): executive summary-a report of the American College of Cardiology/American Heart Association Task Force on Practice Guidelines (committee to revise the 1993 guidelines for percutaneous transluminal coronary angioplasty) endorsed by the Society for Cardiac Angiography and Interventions. Circulation. 2001;103: 3019-3041.

14. Berman DS, Kiat H, Friedman JD, et al. Separate acquisition rest thallium-201/ stress technetium- $99 \mathrm{~m}$ sestamibi dual-isotope myocardial perfusion single- photon emission computed tomography: a clinical validation study. J Am Coll Cardiol. 1993;22:1455-1464.

15. Gibbons RJ, Balady GJ, Bricker JT, et al. ACC/AHA 2002 guideline update for exercise testing: summary article-a report of the American College of Cardiology/American Heart Association Task Force on Practice Guidelines (committee to update the 1997 exercise testing guidelines). J Am Coll Cardiol. 2002;40:1531-1540.

16. Klocke FJ, Baird MG, Lorell BH, et al. ACC/AHA/ASNC guidelines for the clinical use of cardiac radionuclide imaging: executive summary - a report of the American College of Cardiology/American Heart Association Task Force on Practice Guidelines (ACC/AHA/ASNC committee to revise the 1995 guidelines for the clinical use of cardiac radionuclide imaging). J Am Coll Cardiol. 2003;42: 1318-1333.

17. Zellweger MJ, Tabacek G, Zutter AW, et al. Evidence for left ventricular remodeling after percutaneous coronary intervention; effect of percutaneous coronary intervention on left ventricular ejection fraction and volumes. Int $J$ Cardiol. 2004;96:197-201.

18. Cerqueira MD, Weissman NJ, Dilsizian V, et al. Standardized myocardial segmentation and nomenclature for tomographic imaging of the heart: a statement for healthcare professionals from the Cardiac Imaging Committee of the Council on Clinical Cardiology of the American Heart Association. Circulation. 2002;105:539-542.

19. Pfisterer M, Brunner-La Rocca HP, Buser PT, et al. Late clinical events after clopidogrel discontinuation may limit the benefit of drug-eluting stents: an observational study of drug-eluting versus bare-metal stents. J Am Coll Cardiol. 2006;48:2584-2591.

20. Cutlip DE, Windecker S, Mehran R, et al. Clinical end points in coronary stent trials: a case for standardized definitions. Circulation. 2007;115:2344-2351. 